\title{
Factores que impiden el empoderamiento económico de la mujer chihuahuense
}

\section{Factors that prevent the economic empowerment of chihuahuense women}

\author{
MARTÍNEZ-TORRES, Elsy Denise $\dagger$, ORNELAS-BENÍTEZ, Olinda, ESTRADA-PEREA, Jorge Luis \\ y VALLES-BACA, Herik Germán
}

Centro Universitario Parral de la Universidad Autónoma de Chihuahua, General Jesús Lozoya Solís s/n km 1. Col Almanceña

ID $1^{\mathrm{er}}$ Autor: Elsy Denise, Martínez-Torres

ID $1^{\text {er }}$ Coautor: Olinda, Ornelas-Benítez

ID $2^{\text {do }}$ Coautor: Jorge Luis, Estrada-Perea

ID $3^{\text {er }}$ Coautor: Herik Germán, Valles-Baca

DOI: $10.35429 / J O C S .2019 .18 .6 .28 .36$

Recibido: 09 de Enero, 2019; Aceptado 26 de Marzo, 2019

\section{Resumen}

En las últimas décadas la participación de la mujer en todos los ámbitos es cada vez mayor, en materia del ámbito laboral las estadísticas demuestran este incremento, sin embargo, también se ha demostrado que en México el porcentaje de prácticas discriminativas hacia la mujer están presentes en el mercado laboral, lo que ha llevado a generar altos índices de discriminación salarial y una menor probabilidad de obtener puestos mejor remunerados. Por la importancia de este tema, en este estudio se presentan los principales factores que impiden el empoderamiento de la mujer Chihuahuense, mediante una metodología estadística descriptiva, centrando su análisis en el estudio de las características entre hombres y mujeres al incorporarse al mercado laboral y como se ven reflejadas en la remuneración salarial, con la finalidad de comprobar si este fenómeno se debe a factores sociodemográficos o a un problema de perspectiva de género, y así mostrar el panorama actual al que se enfrentan las mujeres en el estado de Chihuahua.

Discriminación, Perspectiva de género, Empoderamiento

\begin{abstract}
In recent decades, the participation of women in all movements is increasing, in the field of labor statistics affected by this increase, however, the percentage of discriminatory practices towards women has also been detected in Mexico in the labor market, which has managed to generate high rates of wage discrimination and a lower probability of obtaining better paid positions. Due to the importance of this issue, this study presents the main factors that impede the empowerment of Chihuahua women, through a descriptive statistical methodology, focusing their analysis on the study of the characteristics between men and women when entering the labor market and how They are reflected in the salary remuneration, with the way of knowing if this phenomenon is due to sociodemographic factors or a gender perspective problem, and thus show the current panorama faced by women in the state of Chihuahua.
\end{abstract}

Teaching mediation, emotional intelligence, positive emotions

Citación: MARTÍNEZ-TORRES, Elsy Denise, ORNELAS-BENÍTEZ, Olinda, ESTRADA-PEREA, Jorge Luis y VALLESBACA, Herik Germán. Factores que impiden el empoderamiento económico de la mujer chihuahuense. Revista de Sociología Contemporánea. 2019. 6-18: 28-36.

\footnotetext{
$\dagger$ Investigador contribuyendo como primer autor.
} 


\section{Introducción}

Como primicia se muestra la sustentación teórica de las causas y consecuencias a lo largo de la historia de cómo la desigualdad de género ha tenido un impacto significativo en el empoderamiento económico de la mujer, tomando como elemento esencial las brechas salariales, la discriminación en la asignación de puestos, los roles de género asignados por conductas sociales y culturales, así como la verificación de los programas implementados a favor de la igualdad social.

Para el análisis del panorama actual acerca del empoderamiento de la mujer y la promoción de la igualdad, se presenta un análisis de lo general a lo particular, iniciando a nivel global hasta concluir con el estado de Chihuahua; examinando las organizaciones, asociaciones civiles, programas gubernamentales y políticas públicas enfocados a la erradicación de la discriminación social y laboral.

Los datos utilizados en esta investigación fueron tomados del INEGI, de la Encuesta Nacional de Ocupación y Empleo (ENOE) y de la Encuesta Nacional de Ingresos y Gastos del Hogar (ENIGH), basados a la última publicación de información según la parcialidad de cada una de las encuestas, cabe mencionar que los datos recabados de cada una de ellas fueron tomados en cuenta debido a los factores más importantes a analizar dentro del empoderamiento económico de la mujer.

Esta investigación se inclina a una metodología cuantitativa y un análisis cualitativo de la información recabada, para lograr identificar los principales factores que impiden el empoderamiento económico de la mujer Chihuahuense.

\section{Revisión de la literatura}

Empoderamiento de la mujer.

En los últimos años se ha tomado como sujeto de estudio las desigualdades socioculturales y económicas entre hombres y mujeres, y se asemeja a un tema actual, sin embargo, este tema data de décadas atrás, tomando como punto de partida los movimientos feministas ocurridos en los años 70 y 80 en América Latina y el Caribe donde el principal objetivo fue la búsqueda del llamado empoderamiento, relacionado este concepto a la toma de "poder", haciendo referencia principalmente en un camino hacia una mayor identidad y la capacidad de la toma de decisiones que ayudarán a generar un impacto dentro de los temas económicos, políticos y sociales.

De este surgimiento, en la cuarta Conferencia Mundial sobre la Mujer desarrollada en Beijing en 1995, se pone en manifiesto promover los objetivos de igualdad, desarrollo y paz para todas las mujeres del mundo, con intereses favorecedores para toda la humanidad, así mismo se reconoció los avances que se habían alcanzado sin omitir que no se han logrado progresos homogéneos, por la existencia de las desigualdades entre hombres y mujeres, por lo que dentro de esta conferencia se dio a conocer el concepto del empoderamiento de la mujer refiriéndose a este con la siguiente declaración: "La potenciación del papel de la mujer y la plena participación de la mujer en condiciones de igualdad en todas las esferas de la sociedad, incluidos la participación en los procesos de adopción de decisiones y el acceso al poder, son fundamentales para el logro de la igualdad, el desarrollo y la paz" (Párrafo 13)

Dados estos hechos el concepto de empoderamiento de la mujer tomo fuerza y formalizó varios análisis para llevar este concepto no como la toma de poder de la mujer, por lo contrario, darle el sentido de igualdad de toma de decisiones y autonomía, para establecer planes de inclusión en condiciones de participación política, económica y áreas de desarrollo. 
John Durston, oficial de asuntos sociales de la División Social de la CEPAL en el 2000, analiza en su teoría de capital social la importancia del empoderamiento de los sectores sociales excluidos, como una estrategia para la superación de la pobreza y desarrollo, poniendo como punto central la buena aplicación del concepto de empoderamiento, que es la esencia de la autogestión, dando así la oportunidad de fortalecer las posibilidades ya existentes de contribuir al crecimiento y desarrollo económico y social. Por lo tanto, tomando en cuenta que, desde hace décadas hasta la actualidad, la mujer sigue siendo excluida de varias esferas sociales, su incorporación en las mismas resulta tener un impacto positivo ante la transformación de los países y el comportamiento entre hombres y mujeres con una visión de igualdad que contribuya para abrir paso a la generación de nuevas ideas y una mayor calidad de vida. " $E l$ empoderamiento de las mujeres llevaría a la liberación de los hombres de los sistemas de valores falsos y de las ideologías de opresión. Llevaría a una situación en la que cada uno sea más integro, prescindiendo del género y utilizando al máxima su potencial en la construcción de una sociedad más humana para todos." (Akhtar, 1992)

Es necesario mencionar que el empoderamiento femenino no solo por ser algo que es necesario $y$ efectivo surge espontáneamente, por el contrario, este lleva un proceso en el cuál se incluyen vertientes que incluyen factores internos y externos; al hablar de los factores internos se hace referencia al reconocimiento por parte de las mujeres que aquellos roles sociales que fueron establecidos por culturas y costumbres en donde la discriminación tanto en actividades económicas, religiosas, toma de decisiones y ausencia de realizar propuestas o simplemente exponer sus ideas, solo reflejan una falla social, jurídica y económica que tiene que ser remplazado por un cambio de conciencia y autoimagen, las mujeres tienen que estar convencidas de sus derechos innatos a la igualdad, la dignidad y la justicia. (Batliwala, 1997)
Dentro de los factores externos se encuentra el reconocimiento de la sociedad del valor de los derechos de igualdad entre hombres y mujeres, a pesar de los avances sobre este tema, la desigualdad en la sociedad por cuestión de género está presente y los roles marcados por sexo impiden el aceleramiento de sociedades con una visión tejedora de hilos de capacidades, habilidades, responsabilidades indistintas de género que fijen intereses en conjunto con objetivos de bienestar social.

\section{México.}

El empoderamiento de la mujer en

En relación de los avances en materia de empoderamiento de la mujer en México, las estadísticas muestran el avance en cuestión de igualdad de oportunidades en la mayoría de las áreas encaminadas al crecimiento económico, jurídico y cultural, esto se debe a que se dio paso a crear bases jurídicas y normativas en el país que garantizan la igualdad de género. El Plan de Desarrollo contiene una estrategia transversal para incorporar la igualdad sustantiva en los programas sectoriales, reflejado principalmente en el Programa Nacional para la Igualdad de Oportunidades y No Discriminación contra las Mujeres 2013-2018 (PROIGUALDAD), que está dirigido por el Instituto Nacional de las Mujeres. (ONU Mujeres, 2015)

A pesar de contar con bases jurídicas en el país para erradicar este problema social, el camino para llegar a una total igualdad de género aun presenta discrepancias que representan barreras para lograrlo.

Es por esto que varias organizaciones se han solidarizado con México para fungir como apoyo y enfrentar este fenómeno con buenas estrategias basadas en una normatividad $y$ objetivos bien definidos. La Organización de las Naciones Unidas y México mantienen relaciones cooperativas por más de 68 años, en las cuales se enfocan en diversos ámbitos y uno de ellos es la igualdad de género y el empoderamiento de las mujeres; dentro de este tema, se mantienen relaciones con diferentes sectores sociales, niveles de gobierno, sector privado y sector académico, con la finalidad de crear lazos y convenios de cooperación para potencializar las fuerzas ya existentes en cada área que permitan la inclusión de todos los participantes de la sociedad. 
Con este fundamento el Programa Nacional para la Igualdad de Oportunidades y No Discriminación contra las Mujeres 20132018, en conjunto con la CEDAW, también conocida como la Carta de los Derechos de las Mujeres, establecieron seis prioridades nacionales que guían los esfuerzos gubernamentales para el logro de la igualdad de género en el ámbito nacional y local.

1. Igualdad sustantiva entre mujeres y hombres.

2. Erradicación de la violencia contra las mujeres $\mathrm{y}$ acceso a la justicia.

3. Empoderamiento económico de las mujeres.

4. Desarrollo social y bienestar de la mujer.

5. Entornos seguros y sensibles al género.

6. Incorporación transversal de la perspectiva de género. (ONU Mujeres 2015)

Mediante estas seis prioridades es posible romper las barreras que se presentan en las esferas sociales, sin embargo, el problema persistente es un México con un cabio de conciencia como ciudadanos al respetar los derechos de igualdad, ya que el Comité de la CEDAW en el 2012, realizó una revisión de los avances que se han registrado y formulo una serie de recomendaciones en materia de armonización legislativa, violencia contra las mujeres, feminicidios, trata de personas, derechos a la educación y empleo de las mujeres, con la finalidad de acelerar la erradicación de casos de discriminación, y mediante esta serie de recomendaciones se confirma de la igualdad de género va más allá del contar con una base jurídica en el país de la materia.( México ante la CEDAW 2012)

Por años la participación de la ONU en temas de desarrollo ha estado presente en México, y prioritariamente toma aquellas áreas en donde la desigualdad entre hombres y mujeres son estructurales, una de las áreas es la representada por la Autonomía y Empoderamiento Económico, dentro de esta sección se realizó un marco normativo enfocado a derechos económicos y sociales de las mujeres, en donde se precisan derechos, empleo e ingresos, protección social y diálogo social, que se desarrollaron en los convenios internacionales de la OIT.
A continuación, se presentan las principales iniciativas a favor de la autonomía y el empoderamiento económico.

\begin{tabular}{|c|c|c|}
\hline PARTICIPANTES & INICITIVA & OBJETIVO \\
\hline $\begin{array}{l}\text { ONU Mujeres, } \\
\text { CEPAL, } \\
\text { INMUJERES e INEGI }\end{array}$ & $\begin{array}{lr}\text { Orientadas a la } \\
\text { producción } \\
\text { uso } \\
\text { estadística } \\
\text { acerca } \\
\text { Trabajo } \\
\text { remunerado y } \\
\text { no remunerado , } \\
\text { Uso del tiempo. }\end{array}$ & $\begin{array}{l}\text { Fungir como un } \\
\text { espacio de } \\
\text { gestión de } \\
\text { conocimiento y } \\
\text { reflexión, y de } \\
\text { trabajo técnico- } \\
\text { metodológico } \\
\text { para medir el } \\
\text { trabajo } \\
\text { doméstico no } \\
\text { remunerado e } \\
\text { integrarlo a las } \\
\text { Cuentas } \\
\text { Satélite. }\end{array}$ \\
\hline $\begin{array}{l}\text { OIT, ONU Mujeres y } \\
\text { CONAPRED }\end{array}$ & $\begin{array}{l}\text { Visualizar la } \\
\text { discriminación } \\
\text { de las que son } \\
\text { objeto las } \\
\text { trabajadoras } \\
\text { domesticas }\end{array}$ & $\begin{array}{l}\text { Dar } \\
\text { cumplimiento a } \\
\text { sus derechos } \\
\text { laborales y } \\
\text { económicos }\end{array}$ \\
\hline $\begin{array}{l}\text { Centro de Comercio } \\
\text { Internacional (ITC) y } \\
\text { el Instituto Nacional } \\
\text { de Apoyo a la } \\
\text { Economía Social. }\end{array}$ & $\begin{array}{lr}\text { Incrementar } & \text { la } \\
\text { demanda } & \text { de } \\
\text { bienes } & y \\
\text { servicios } & \\
\text { provistos } & \text { por } \\
\text { mujeres } & \\
\text { empresarias. } & \end{array}$ & $\begin{array}{l}\text { Desarrollar las } \\
\text { capacidades de } \\
\text { las mujeres para } \\
\text { exportar y } \\
\text { desarrollar las } \\
\text { capacidades } \\
\text { institucionales } \\
\text { para ofrecer } \\
\text { mejores } \\
\text { servicios para } \\
\text { las mujeres que } \\
\text { atienden. }\end{array}$ \\
\hline ONU Mujeres & $\begin{array}{l}\text { Derechos de las } \\
\text { trabajadoras } \\
\text { migrantes en la } \\
\text { frontera sur de } \\
\text { México. }\end{array}$ & $\begin{array}{l}\text { Promoción y } \\
\text { protección de } \\
\text { los derechos } \\
\text { laborales y } \\
\text { humanos de las } \\
\text { trabajadoras } \\
\text { migrantes. }\end{array}$ \\
\hline
\end{tabular}

Tabla 1 Elaboración propia con datos de ONU Mujeres 2015

Chihuahua, panorama actual del empoderamiento económico de la mujer.

El empoderamiento económico y social es sin duda una estrategia con un impacto significativo para el desarrollo de las comunidades, por lo tanto, en el estado de Chihuahua se ha tomado como un punto de partida el análisis estadístico de bases de datos tomadas de las últimas publicaciones realizadas por el INEGI que son significativas para esta investigación. El estado de Chihuahua en materia de empoderamiento económico se ha reconocido un avance, sin embargo, la meta de un panorama igualitario aún tiene retos por cumplir.

MARTÍNEZ-TORRES, Elsy Denise, ORNELAS-BENÍTEZ, Olinda, ESTRADA-PEREA, Jorge Luis y VALLES-BACA, Herik Germán. La mediación docente para fomentar la educación emocional de los estudiantes de una Universidad Pública de Toluca. Revista de Sociología Contemporánea. 2019 


\section{Análisis de los datos}

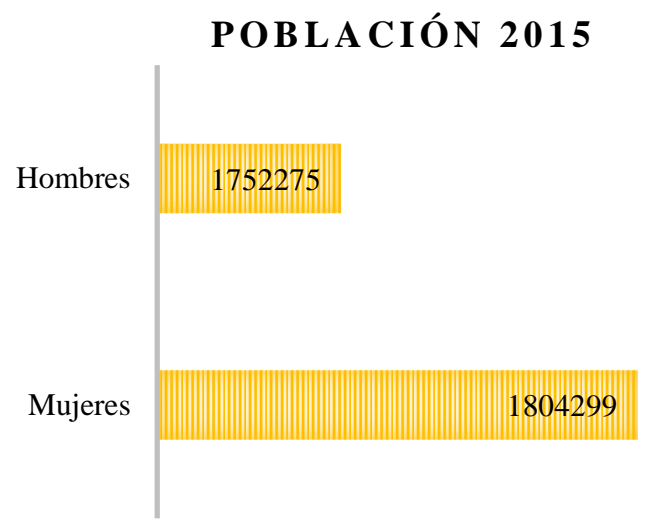

172000017400001760000178000018000001820000

Gráfico 1 Población

Fuente: elaboración propia, con datos de INEGI 2015

La población para 2015 en el estado de Chihuahua fue de 1804299 mujeres y 172275 hombres.

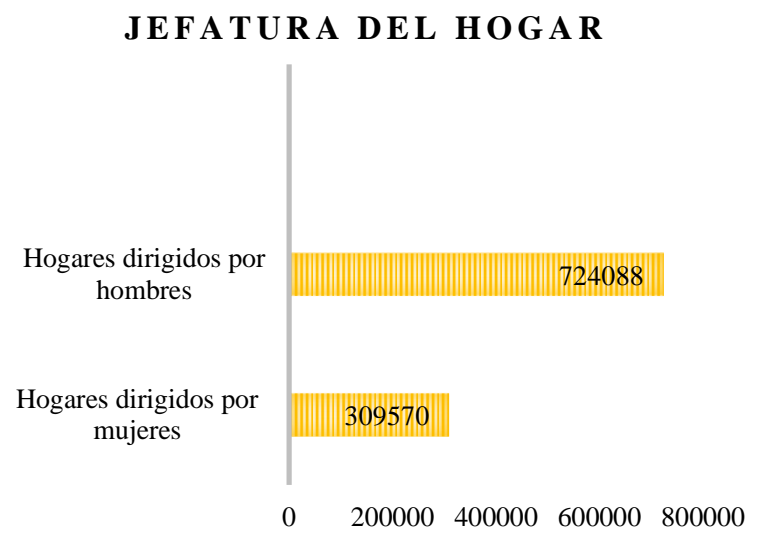

Gráfico 2 Jefatura del hogar

Fuente: elaboración propia, con datos de INEGI 2015

El $30 \%$ de los hogares en el estado de Chihuahua son dirigidos por una mujer.

\section{POBLACIÓN ECONOMICAMETE A C T IVA / O CUPADA}

(MUJERES 15 AÑ OS Y MÁS, 2017 )

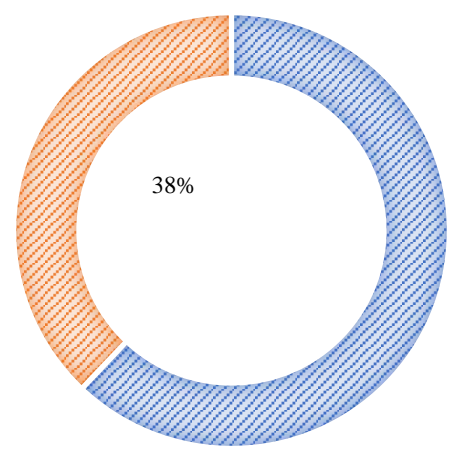

Gráfico 3 Población Económicamente activa/ocupada Fuente: elaboración propia, con datos de INEGI y ENOE 2017

\section{POBLACIÓN ECONOMICAMETE \\ ACTIVA/OCUPADA \\ (HOMBRES 15 A ÑOS Y MÁS, 2017 )}

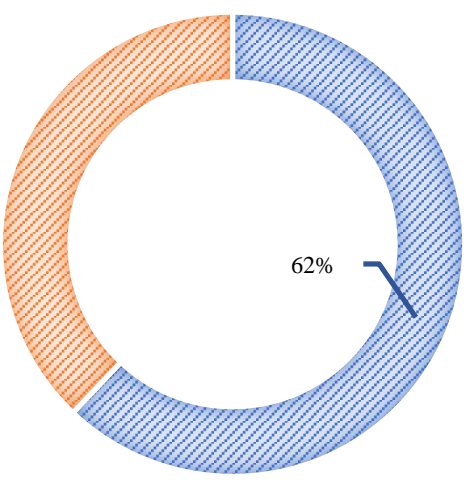

Gráfico 4 Población Económicamente activa/ocupada Fuente: elaboración propia, con datos de INEGI y ENOE 2017

El 38\% de las mujeres representan a la población económicamente ocupada, mientras que el $62 \%$ de la población económica ocupada está conformado por hombres.

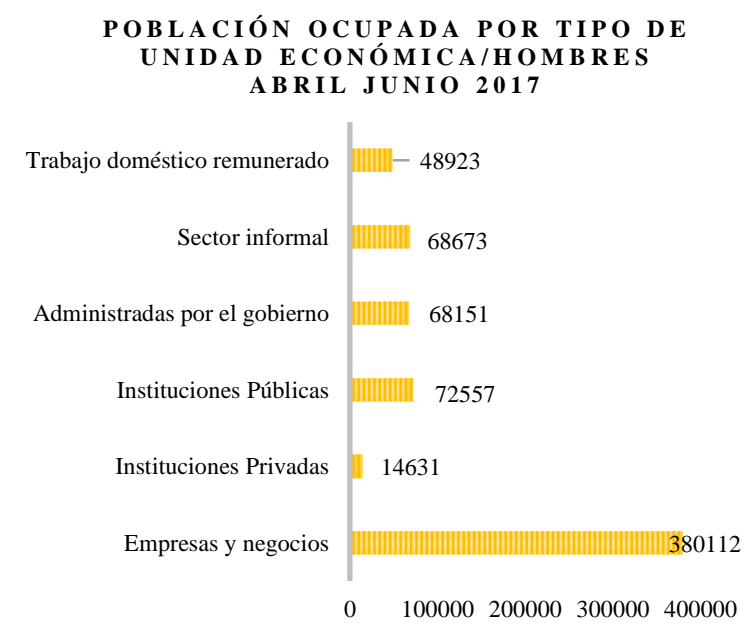

Gráfico 5 Población ocupada por tipo de unidad económico/hombres

Fuente: elaboración propia, con datos de INEGI y ENOE 2017

De la población ocupada por los hombres, se encuentran mayormente ubicados en empresas y negocios, seguidos por instituciones públicas, sector informal, unidades administradas por el gobierno, trabajo doméstico remunerado $y$ en última instancia en instituciones privadas.
MARTÍNEZ-TORRES, Elsy Denise, ORNELAS-BENÍTEZ, Olinda, ESTRADA-PEREA, Jorge Luis y VALLES-BACA, Herik Germán. La mediación docente para fomentar la educación emocional de los estudiantes de una Universidad Pública de Toluca. Revista de Sociología Contemporánea 2019 
Marzo, 2019 Vol.6 No.18 28-36

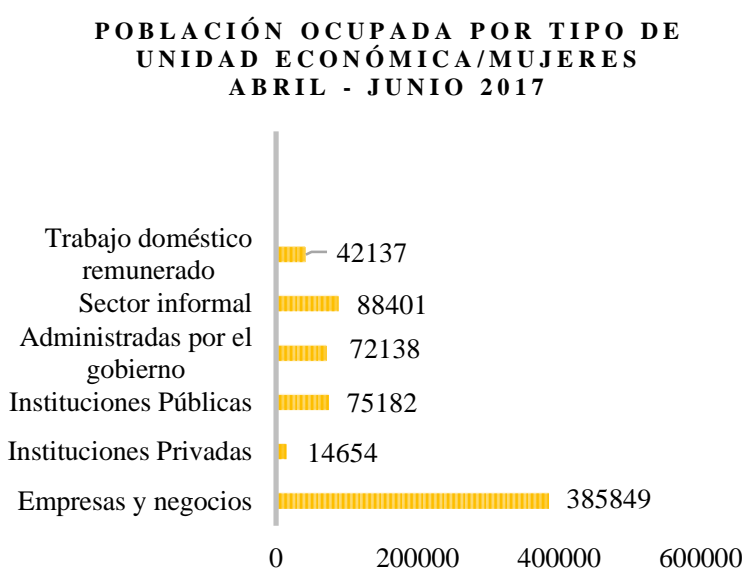

Gráfico 6 Población ocupada por tipo de unidad económico/hombres

Fuente: elaboración propia, con datos de INEGI y ENOE 2017

De la población ocupada por las mujeres, se encuentran mayormente ubicadas en empresas y negocios, seguidos por unidades del sector informal, instituciones públicas, unidades administradas por el gobierno, trabajo doméstico remunerado $y$ en última instancia en instituciones privadas.

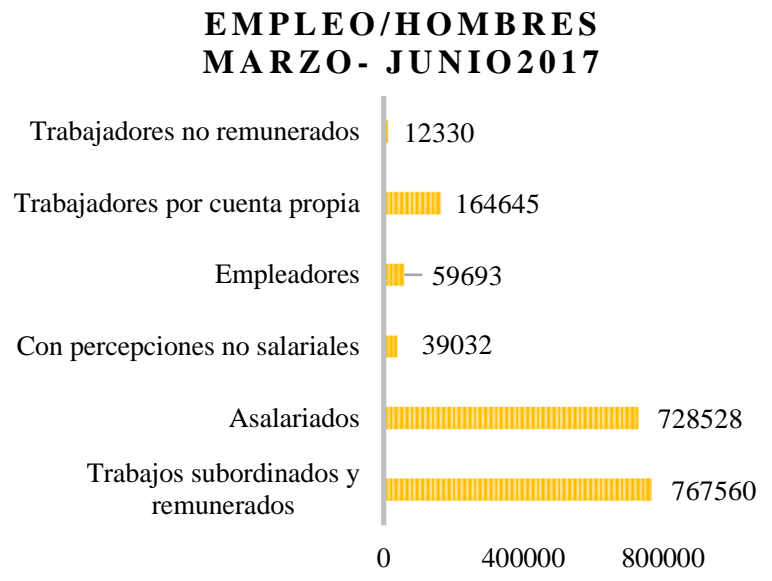

Gráfico 7 Población Económicamente activa/ocupada Fuente: elaboración propia, con datos de INEGI y ENOE 2017

Los hombres se encuentran empleados con mayor frecuencia en trabajos subordinados y remunerados, seguidos de trabajos asalariados, trabajadores por su cuenta, empleadores, con percepciones no salariales y trabajos no remunerados.
EMPLEO/MUJERES

MARZO - JUNIO 2017

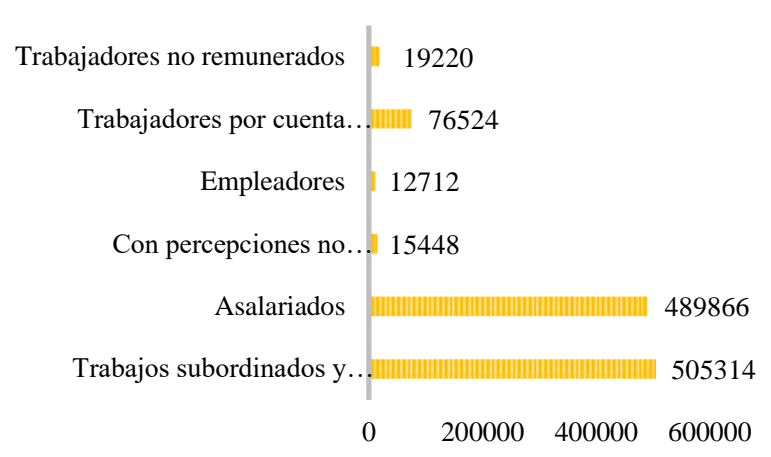

Gráfico 8 Población Económicamente activa/ocupada Fuente: elaboración propia, con datos de INEGI y ENOE 2017

Las mujeres se encuentran empleadas con mayor frecuencia en trabajos subordinados y remunerados, seguidos de trabajos asalariados, trabajadores por su cuenta, con percepciones no salariales, empleadores y trabajos no remunerados.
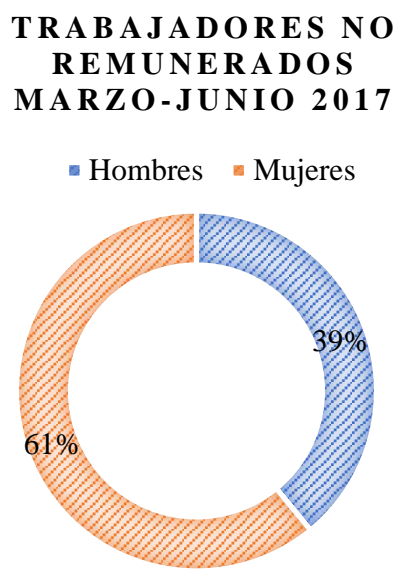

Gráfico 9 Población Económicamente activa/ocupada Fuente: elaboración propia, con datos de INEGI y ENOE 2017

De la población económicamente activa el $61 \%$ está compuesta por los hombres, mientras que el $39 \%$ lo ocupan las mujeres. 
HOMBRES OCUPADOS POR NIVEL DE INGRESO MARZO-JUNIO 2017

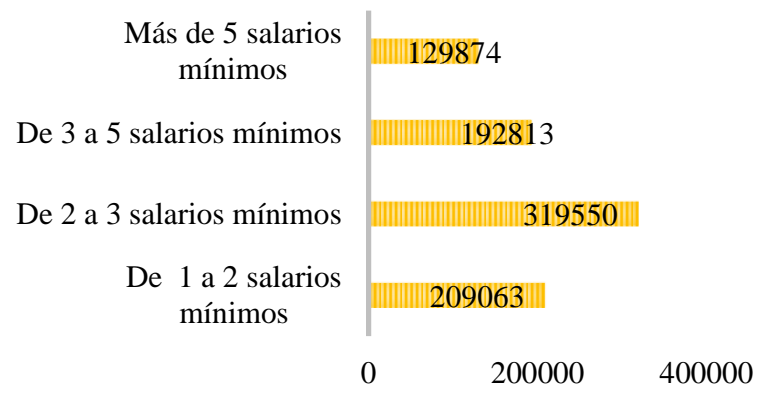

Gráfico 10 Población Económicamente activa/ocupada Fuente: elaboración propia, con datos de INEGI y ENOE 2017

El nivel de ingreso de los hombres es en su mayoría de 2 a 3 salarios mínimos, y solo 129874 reciben más de 5 salarios mínimos.

\section{MUJERES OCUPADAS POR \\ NIVEL DE INGRESO \\ MARZO-JUNIO 2017}

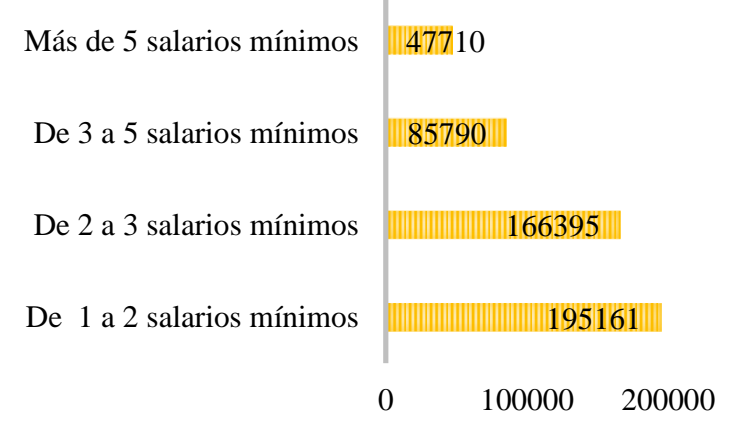

Gráfico 11 Población Económicamente activa/ocupada Fuente: elaboración propia, con datos de INEGI y ENOE 2017

El nivel de ingreso de las mujeres es en su mayoría de 2 a 3 salarios mínimos, y solo 47710 reciben más de 5 salarios mínimos.

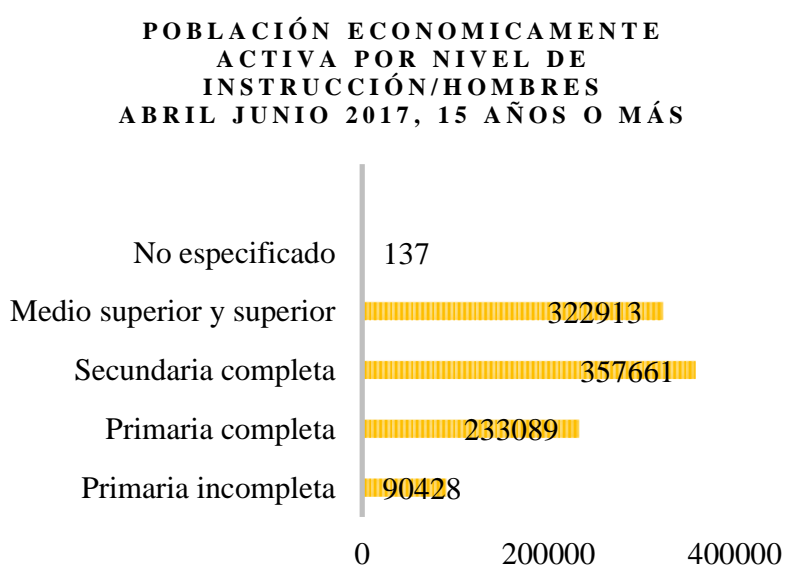

Gráfico 12 Población Económicamente activa/ocupada por nivel de instrucción/hombres

Fuente: elaboración propia, con datos de INEGI y ENOE 2017
De la población económicamente activa los hombres tiene el siguiente nivel de instrucción, 357661 cuentan con secundaria completa, 322913 con medio superior o superior, 233089 con primaria completa y 90428 con primaria incompleta.

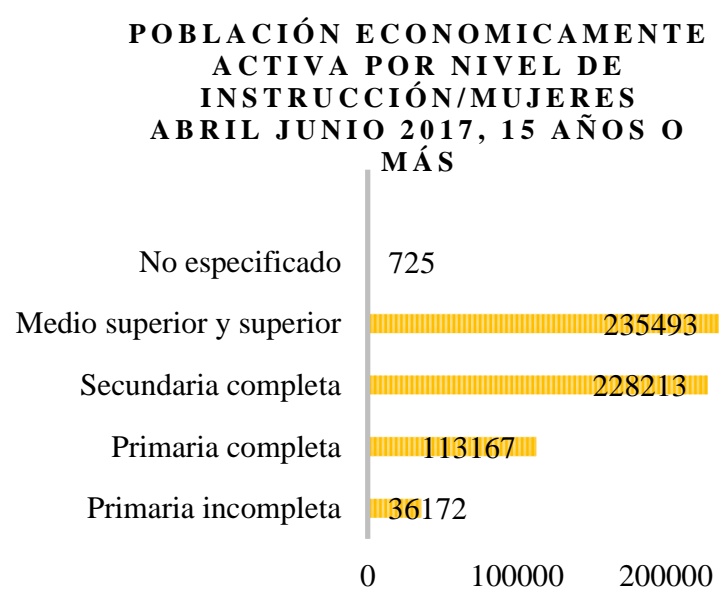

Gráfico 13 Población Económicamente activa/ocupada por nivel de instrucción/mujeres

Fuente: elaboración propia, con datos de INEGI y ENOE 2017

De la población económicamente activa las mujeres tiene el siguiente nivel de instrucción, 235493 cuentan con medio superior y superior, 228213 con secundaria completa, 113167 con primaria completa y 36172 con primaria incompleta.

VIOLENCIA LABORAL CONTRA LAS MUJERES 2016 MUJERES OCUPADAS DE 15 AÑ̃S O MÁS

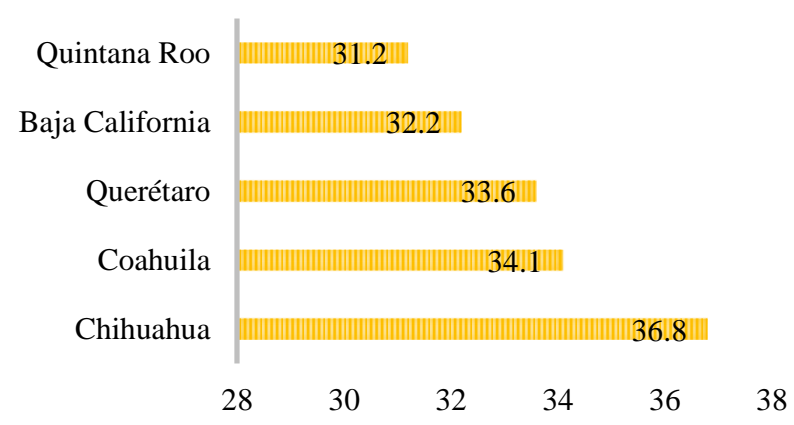

Gráfico 14 Violencia laboral contra la mujer Elaboración propia, con datos de ENDIREH 2017

A nivel nacional los estados con mayor violencia laboral están representados en la figura 8, siendo Chihuahua el estado con mayor índice de violencia laboral, tomando en cuenta los principales tipos de violencia: emocional, física, sexual y por discriminación.

MARTÍNEZ-TORRES, Elsy Denise, ORNELAS-BENÍTEZ, Olinda, ESTRADA-PEREA, Jorge Luis y VALLES-BACA, Herik Germán. La mediación docente para fomentar la educación emocional de los estudiantes de una Universidad Pública de Toluca. Revista de Sociología Contemporánea. 2019 
CONDICIÓN DE TRABAJO NO

REMUNERADO/HOMBRES

MARZO 2015, 12 AÑOS Y MÁS

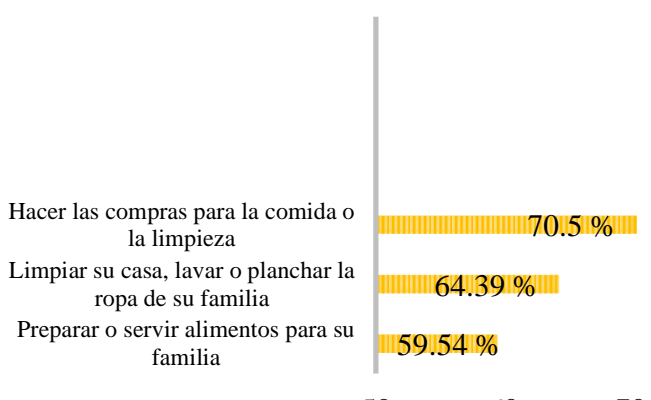

$\begin{array}{llll}50 & 60 & 70 \quad 80\end{array}$

Gráfico 15 Condiciones de trabajo no remunerado/Hombres

NOTA: la suma de los porcentajes puede ser mayor a $100 \%$ debido a las personas que realizan más de una actividad sin pago

Fuente: elaboración propia, con datos de INEGI Encuesta Intercensal 2017

En el campo del trabajo no remunerado el hombre desempeña las siguientes actividades $70.5 \%$ hacer las comprar para la comida o la limpieza, $64.39 \%$ a limpiar su casa, lavar o planchar la ropa de su familia y el $59.54 \%$ a preparar o servir alimentos para su familia.

CONDICIÓN DE TRABAJO NO REMUNERADO/MUJERES

MARZO 2015, 12 A ÑOS Y MÁS

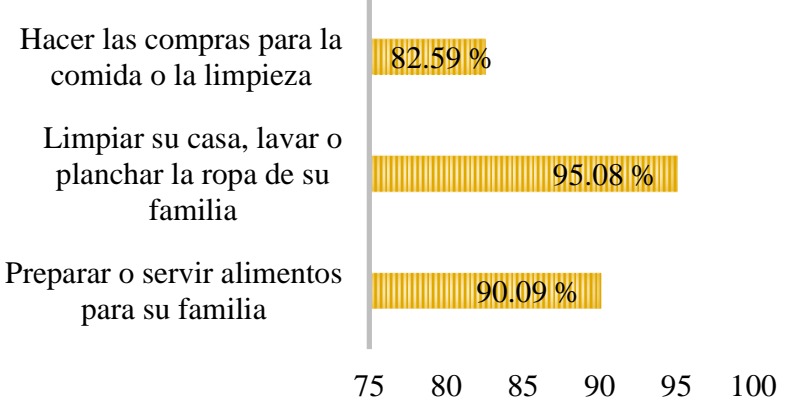

Gráfico 16 Condiciones de trabajo no remunerado/Mujeres

NOTA: *La suma de los porcentajes puede ser mayor a $100 \%$ debido a las personas que realizan más de una actividad sin pago.

Fuente: elaboración propia, con datos de INEGI Encuesta Intercensal 2017

En el campo del trabajo no remunerado la mujer desempeña las siguientes actividades 95.08\% a limpiar su casa, lavar o planchar la ropa de su familia, $90.09 \%$ a preparar o servir alimentos para su familia y el $82.59 \%$ hacer las compras para la comida o la limpieza.

\section{Conclusiones}

Una vez realizado un análisis teórico, sustentado con bases estadísticas, se concluye que efectivamente la desigualdad de género sigue siendo un reto para el estado de Chihuahua, es necesario mencionar que los esfuerzos por erradicar este fenómeno y promocionar la igualdad ha tenido avances, sin embargo, el camino aún es largo y los retos siguen surgiendo a medida que las características del entorno actual se van modificando.

Se demuestra que el estado de Chihuahua cuenta con políticas públicas y programas gubernamentales enfocados a la igualdad de género, con objetivo de generar estrategias que aminoren las prácticas de discriminación en la sociedad y en el mercado laboral.

Una vez analizados estos programas y verificada la estadística de los datos más relevantes a examinar acerca de desigualdad de género, se puede demostrar que, a pesar de las propuestas y normas establecidas, los resultados nos indica que la brecha salarial por género está presente. Con esta información podemos concluir que los principales factores para el empoderamiento económico de la mujer Chihuahuense son los siguientes:

Aspectos culturales que impiden que la mujer pueda acceder a puestos de mayor jerarquía en el mercado laboral, en donde se supone que el hombre puede desempeñar un puesto de mayor responsabilidad ya que puede dedicar más de su tiempo a la vida laboral, en comparación al de una mujer.

Los roles asignados por aspectos conductuales y culturales provocan que para una empresa pueda generar altos costos al contratar a una mujer, ya que, la mujer tiene "asignadas" algunas actividades por el hecho de ser mujer, como cuidar a los hijos, tareas del hogar, cuidados de la salud para su familia, maternidad, cuidados al adulto mayor, lo que representa para las empresas incapacidades, ausencias en jornadas de trabajo, cuidados maternos. 
A pesar de vivir en un mundo en constante cambio, tecnologías, innovación, ciencia y emprendimiento social, hace falta un cambio de conciencia dirigida a una igualdad de oportunidades, a una mirada hacia una sociedad con una sólida meta de crecimiento económico y social basado en la cooperación de género, en la inclusión de habilidades y aptitudes.

En materia empresarial es necesario que los compromisos se conviertan en acciones, para que los cumplimientos de las políticas gubernamentales puedan ser medibles y cuantificables, con el objetivo de un crecimiento económico a nivel estatal y que las cifras por violencia laboral puedan erradicarse.

\section{Referencias}

Declaración y Plataforma de Acción Beijing, septiembre, 1995.

El significado del empoderamiento de las mujeres: nuevos conceptos desde la acción.

Srilatha Batliwala, 1997.

La ONU en acción para la Igualdad de Género en México 2015.

Anuario Estadístico y geográfico de Chihuahua 2017/INEGI.

Resultados de la encuesta nacional sobre la dinámica de las relaciones en los hogares (ENDIREH) 2016. 